\title{
Apoyo de las relaciones públicas a los programas de responsabilidad social en las empresas del sector eléctrico colombiano ${ }^{1}$
}

\section{Ángela Preciado-Hoyos ${ }^{2}$}

Recibido: 2013-09-12

Enviado a pares: 2013-09-13
Aprobado por pares: 2013-11-29

Aceptado: 2014-02-23

DOI: $10.5294 /$ pacla.2015.18.1.10

\section{Para citar este artículo / To reference this article / Para citar este artigo}

Preciado-Hoyos, A. Marzo de 2015. Apoyo de las relaciones públicas a los programas de responsabilidad social en las empresas del sector eléctrico colombiano. Palabra Clave 18(1), 239-257. DOI: 10.5294/pacla.2015.18.1.10

\section{Resumen}

Las relaciones públicas y la responsabilidad social empresarial comparten perspectivas para gestionar las interacciones entre la organización y sus grupos de interés. Sin embargo, desde algunas visiones teóricas y aplicadas, las relaciones públicas han tenido una connotación negativa, de apariencia y ocultamiento de información. En este artículo se presentan los resultados de una investigación de tipo exploratorio, orientada a determinar cuál es el rol que cumplen las relaciones públicas en la gestión de los programas de responsabilidad social de un grupo de empresas del sector eléctrico colombiano. Los resultados indican que entre los consultados no se maneja un concepto unificado sobre las relaciones públicas y se presentan casos en los que la visión que se tiene sobre ellas es negativa.

1 Este artículo es resultado del proyecto de investigación "El papel de las relaciones públicas en los programas de responsabilidad social de las empresas del sector energético colombiano” (COM47-2010), financiado con recursos de la Dirección de Investigación y la Facultad de Comunicación de la Universidad de La Sabana.

2 Universidad de La Sabana. Facultad de Comunicación, Colombia. angela.preciado@unisabana.edu.co 


\title{
Palabras clave
}

Relaciones públicas, responsabilidad social, energía eléctrica, medio ambiente, proceso de comunicación (Fuente: Tesauro de la Unesco).

\section{Support of Public Relations to the Social Responsibility Programs within the Companies of the Colombian Electric Power Sector}

\begin{abstract}
Public relations and corporate social responsibility share perspectives in managing the interactions between the organization and its stakeholders. However, from the standpoint of some of the theoretical and applied insights, public relations have had a negative connotation of appearance and hiding of information. In this paper we present the findings of an exploratory-type research, aimed at determining what role public relations play in the management of social responsibility programs of a group of companies within the Colombian electric power industry. The findings indicate that, among the respondents, no unified concept is being handled on what public relations are, and cases were found where the point of views regarding such social responsibility programs is negative.
\end{abstract}

\section{Keywords}

Public relations, social responsibility, electric power, environment, communication process (Source: UNESCO Thesaurus). 


\section{Apoio das relações públicas aos programas de responsabilidade social nas empresas do setor elétrico colombiano}

\section{Resumo}

As relações públicas e a responsabilidade social empresarial compartilham perspectivas para administrar as interações entre a organização e seus grupos de interesse. No entanto, a partir de algumas visões teóricas e aplicadas, as relações públicas tiveram uma conotação negativa, de aparência e ocultação de informação. Neste artigo apresentam-se os resultados de uma pesquisa de tipo exploratório que visa determinar qual é o papel das relações públicas na gestão dos programas de responsabilidade social de um grupo de empresas do setor elétrico colombiano. Os resultados indicam que entre os entrevistados não se usa um conceito unificado sobre o que são as relações públicas e se apresentam casos nos quais a visão que se tem sobre elas é negativa.

\section{Palavras-chave}

Relações públicas, responsabilidade social, energia elétrica, meio ambiente, processo de comunicação (Fonte: Tesauro da Unesco). 


\section{Introducción}

En su desarrollo teórico y aplicado, las relaciones públicas han pasado por varios estadios o etapas (Grunig \& Hunt, 1984; Grunig \& Grunig, 1992; Ferrari \& Franca, 2011). Las primeras prácticas asociadas a la profesión generaron percepciones negativas, porque las relaciones se ejercían en una dirección, de la organización hacia sus públicos, y se ajustaba a la concepción de mensajes encaminados a producir en los receptores las actitudes y comportamientos deseados por el emisor.

A partir de trabajos realizados a principios de los años cincuenta del siglo XX (Cutlip \& Center, 1952), empezó a aceptarse en los estudios sobre el área que el entorno es el suprasistema social de las organizaciones y que estas deben adaptarse a él si quieren sobrevivir, así como dialogar con los públicos si desean alcanzar el entendimiento y permanecer en el largo plazo. Desde entonces algunas organizaciones empezaron a investigar la opinión de los grupos de interés; no obstante, muchas procedieron con el ánimo de introducir esfuerzos de persuasión y diseñar programas de comunicación que les aseguraran beneficios (Ferrari \& Franca, 2011).

En trabajos como los de Grunig \& Hunt (1984) se empezaron a aportar criterios y formulaciones que han permitido ir cambiando la orientación de la práctica y, por esa vía, las percepciones que se han tenido sobre la actividad. En su teoría de la excelencia, Grunig (1992) propone abordar las relaciones públicas como una función directiva, entenderlas como relaciones equilibradas, encaminadas a que las organizaciones y sus públicos manejen los mismos niveles de información e influencia en un proceso de interacción. En esta teoría, considerada predominante en el mundo contemporáneo (Jelen, 2008), las relaciones públicas se conciben como un campo de estudios de las ciencias sociales, orientado a buscar el bien común y a interpretar y tratar a los públicos de manera diferenciada. Al proponer la negociación, el consenso y la mediación como ejes de su accionar, este modelo ha sido catalogado como la práctica más ética entre todas las que le han antecedido y ha sido señalado como el más consistente con un ejercicio transparente de la responsabilidad social (Ferrari \& Franca, 2011). El modelo asigna al profesional de las comunicaciones la categoría de estratega cuando integra el grupo directivo de la organización, participa en la 
toma de decisiones haciendo recomendaciones sobre la ejecución de políticas, directrices y planes de comunicación, concibe y formula la planificación global de las relaciones con los grupos de interés, actúa como "analista de escenarios" y hace eco de los puntos de vista de los públicos ante las instancias directivas.

En estudios más actuales se continúa poniendo el acento en el componente social de las relaciones públicas y se resalta su utilidad en la mediación y resolución de conflictos, al igual que en la construcción de relaciones consensuadas, necesarias para mantener el orden social (Van Ruler, Vercic \& Vercic, 2008). En esta línea de pensamiento, Bowen (2005) afirma que los relacionistas públicos deben actuar como la conciencia corporativa, los jueces de la integridad y la honestidad y los consejeros éticos de quienes toman las decisiones más importantes en la organización. Estas funciones se cumplen cuando remplazan la falta de información, la coerción y la intransigencia, con conocimiento, compromiso y elementos que permiten a las organizaciones adaptarse y ajustarse (Clark, 2000; Daugherty, 2001; Sharpe \& Pritchard, 2004; Dawkins, 2004).

Algunos han definido las relaciones públicas como la gestión de las relaciones con personas o grupos que pueden afectar la estabilidad de la organización, o que se ven afectados por sus actuaciones (Grunig \& Hunt, 1984). De manera similar, la teoría del stakeholder, utilizada como referente en numerosas organizaciones para orientar las políticas y prácticas de responsabilidad social, postula que la dirección deberá trabajar para balancear los intereses de los diferentes grupos que tienen alguna relación con la empresa y define a los stakeholders como cualquier grupo o individuo que puede afectar o es afectado por las actuaciones de esta (Freeman, 1984; Freeman \& Phillips, 2002). En la misma teoría, la responsabilidad social es un proceso donde las expectativas de los grupos de interés se correlacionan con las de la empresa, mediante la vinculación y el diálogo.

En la generalidad de estudios sobre comunicación en las organizaciones, las relaciones públicas son la disciplina que ha analizado y discutido más ampliamente los conceptos de relación y públicos (Van Ruler et al., 2008). De ahí que pueda afirmarse que, en términos de formación, los 
profesionales del área cuentan con una ventaja única en las empresas para impactar las estrategias de responsabilidad social (Spangler \& Donalynn 2011). Ahora bien, pese a que numerosos académicos han aportado elementos para que las relaciones públicas se practiquen de manera ética y desde un enfoque estratégico, es preciso hacer notar que existen organizaciones que continúan apoyándose en modelos unidireccionales de comunicación y que hacen énfasis en la propaganda. Esta orientación, que se presenta de manera preponderante entre quienes trabajan para actores de cine y televisión, deportistas, políticos y entidades del sector público, podría explicar que sigan existiendo sospechas sobre la profesión y que se continúe transmitiendo un estereotipo negativo de ella (Cottle, 2003; Kim \& Park, 2011). En muchos casos se mantiene la idea de que las relaciones públicas constituyen esfuerzos de las organizaciones por avanzar en sus propias agendas y las equiparan con la publicity y las relaciones con los medios, lo que para algunos se ha generado, muy probablemente, porque las funciones estratégicas importantes relacionadas con la profesión suelen tener poca visibilidad (White \& Park, 2010).

Para conocer el papel que se concede a las relaciones públicas en la gestión de programas de responsabilidad social, se llevó a cabo una investigación enfocada en un grupo de organizaciones del sector eléctrico colombiano. Es preciso hacer notar que investigaciones realizadas en Colombia muestran que cada vez existe mayor conciencia sobre la importancia de la comunicación en las organizaciones y que en numerosas empresas se contrata agencias externas, especializadas en relaciones públicas, que complementan el trabajo que realizan los profesionales del área que conforman la plantilla de empleados (Preciado \& Guzmán, 2011). Del mismo modo, las agencias internacionales asentadas en el país están pasando por uno de sus mejores momentos, por el número de empresas que se vienen estableciendo y por la facturación del sector, que ascendió a alrededor de 26 millones de dólares entre 2011 y 2012 (Dinero, 2012).

\section{Metodología}

La orientación específica del estudio aplicado ha consistido en conocer si las empresas del sector elegido se apoyan en prácticas de relaciones pú- 
blicas para gestionar la responsabilidad social en lo concerniente al relacionamiento con los grupos de interés y a la manera en que ese apoyo se hace efectivo.

Para responder a esta finalidad, se determinó que la investigación cualitativa era la más adecuada, porque permitía que los profesionales encargados de la comunicación dieran cuenta del enfoque desde el que la organización trabaja estos temas, describieran sus experiencias y comentaran cuál era su consideración particular sobre las cuestiones en estudio. Se optó por realizar entrevistas en profundidad a partir de un cuestionario con preguntas semiestructuradas, porque estas permiten un mayor entendimiento del fenómeno y que los entrevistados puedan explicar y ampliar las respuestas.

El sector eléctrico colombiano fue elegido por la complejidad del negocio, el crecimiento sostenido que ha venido teniendo en los últimos años, los amplios márgenes de ganancia que genera y el riesgo medioambiental que involucra en sus operaciones. Por lo general, los stakeholders que se le asocian demandan mayor transparencia corporativa y diálogo. Del mismo modo, es de esperar que en este sector las estrategias corporativas estén soportadas en prácticas de responsabilidad social por el impacto que sus operaciones pueden tener entre las comunidades y en el medio ambiente, entre otras razones, porque más del $63 \%$ de la producción de energía eléctrica en el país proviene de fuentes hidráulicas (Upme, 2012). Aparte de ello, es un sector muy dinámico. En los últimos diez años se incrementó la inclusión de agentes e inversores privados en las empresas estatales y se desarrollaron nuevos proyectos de infraestructura.

Empresas como EPM, Isagen e ISA tienen negocios dentro y fuera del país, a partir de compras y adquisiciones, o porque obtienen contratos por la vía de las licitaciones públicas. La participación del sector en el PIB nacional total fue del 3,7\% en 2009 (Upme-Ascodis, 2011), y la capacidad instalada para la generación de energía eléctrica era, a julio de 2012, de 14 450 megavatios (Upme, 2012). Colombia es un país que se autoabastece en energía eléctrica, por cuanto la demanda local atendida a julio de 2012 fue de 5026 GW-h (Upme, 2012), lo que ha permitido exportar energía a Venezuela y Ecuador. 
A pesar de que en Colombia el sector está compuesto por alrededor de 40 organizaciones, este es un mercado concentrado, donde las seis empresas más grandes generan, distribuyen y comercializan más del 60\% del total de la energía (Cier, 2011). Se invitó a participar en el estudio a un grupo de 20 organizaciones, en razón de su tamaño, capacidad productiva y, de modo particular, teniendo en cuenta la existencia de profesionales de comunicación en sus plantillas. La participación fue voluntaria, razón por la cual no todas accedieron a atender la entrevista. Dentro del grupo que conforma la muestra están EPM, ISA, Isagen, Gecelca, Celsia, la Empresa Urrá y la Empresa de Energía del Pacífico, EPSA, como se presenta en la tabla 1.

\section{Tabla 1}

\section{Organizaciones consultadas}

\begin{tabular}{|c|c|c|c|c|}
\hline Empresa & $\begin{array}{l}\text { Origen del } \\
\text { capital }\end{array}$ & $\begin{array}{l}\text { Actividades que } \\
\text { realiza en el sector }\end{array}$ & $\begin{array}{c}\text { Ingresos } \\
\text { operacionales en } \\
\text { dólares* }^{*}\end{array}$ & Cargo de las personas entrevistadas \\
\hline ISA & Mixto & $\begin{array}{l}\text { Transmisión } \\
\text { Conexión }\end{array}$ & 4792515571 & Analista de comunicaciones \\
\hline EPM & Público & $\begin{array}{c}\text { Generación } \\
\text { Transmisión } \\
\text { Distribución } \\
\text { Comercialización }\end{array}$ & 4263184228 & Profesional de comunicaciones \\
\hline Celsia & Privado & $\begin{array}{c}\text { Generación } \\
\text { Comercialización }\end{array}$ & 952028105 & Profesional de comunicaciones \\
\hline Isagen & Mixto & $\begin{array}{c}\text { Generación } \\
\text { Comercialización }\end{array}$ & 866165645 & Profesional de comunicaciones \\
\hline EPSA & Privado & $\begin{array}{c}\text { Generación } \\
\text { Transmisión } \\
\text { Distribución } \\
\text { Comercialización }\end{array}$ & 616704,58 & Gerente de comunicaciones \\
\hline Gecelca & Privado & $\begin{array}{c}\text { Generación } \\
\text { Comercialización }\end{array}$ & 675091 & Jefe del área de comunicaciones \\
\hline Empresa Urrá & Mixto & $\begin{array}{c}\text { Generación } \\
\text { Comercialización }\end{array}$ & 94041 & Jefe área de divulgación y prensa \\
\hline
\end{tabular}

* Cifras a 31 de diciembre de 2011. La tasa de cambio del dólar equivalía en esa fecha a \$1942,70 pesos colombianos.

Fuentes: Contraloría General de la República (2012a, 2012b), Grupo EPM (2012), Celsia (2012), Isagen (2012), EPSA (2012), Empresa Urrá (2012). 
Las entrevistas se realizaron entre marzo y mayo de 2012 en Montería, Medellín, Barranquilla y Cali, sedes de las empresas participantes. Cada entrevista tuvo una duración promedio de 90 minutos. El cuestionario contenía 20 preguntas y estaba estructurado en tres partes. En la primera se consultó por el concepto que orienta las acciones de responsabilidad social en cada empresa. En segundo lugar se preguntó por la manera en que los profesionales de la comunicación apoyan las actividades de responsabilidad social. En el último segmento se buscó determinar si las organizaciones se apoyan en las relaciones públicas para definir el marco de relaciones con los grupos de interés.

\section{Hallazgos y discusión}

\section{Concepto de responsabilidad social}

En el grupo de organizaciones consultadas existe unidad de criterio acerca de que la responsabilidad social debe incluir una política de relaciones con todos los grupos de interés y no solo con las comunidades aledañas a las zonas de operación. El concepto de responsabilidad social que se maneja ha evolucionado a lo largo de los años y ha incidido en cómo se estructuran las dependencias e incluso las propias organizaciones. Antes se constituían áreas denominadas "gestión comunitaria" o "gestión social", pero, con la fuerza que fueron tomando las prácticas de responsabilidad social, las entidades dieron relevancia a otros grupos de interés y, al decir de los entrevistados, desde entonces empezaron a definir una manera de establecer compromisos con cada uno de ellos y a orientar la relación, de modo prioritario, a honrar y cumplir los acuerdos pactados.

Hoy en día, en todas las organizaciones existe un área especializada, encargada de liderar el proceso desde este enfoque. Su función básica consiste en dirigir y promover la aplicación de las políticas de responsabilidad social definidas en cada entidad y verificar que se cumplan. Su existencia no descarta que la responsabilidad social sea un compromiso y una práctica transversal a toda la organización.

En Isagen prefieren hablar de la noción de "responsabilidad empresarial" y no de responsabilidad social, porque durante muchos años se pensó 
que la responsabilidad era solo con las comunidades que se asientan en las zonas de operación, por lo general regiones apartadas de los centros urbanos, y acuñar el término social llevaría a seguir perpetuando esa idea.

En EPM, Isagen, EPSA e ISA existen profesionales de la comunicación que apoyan directamente el trabajo de estas áreas y que operan como enlaces con las unidades de comunicación. Al momento de hacer la entrevista, Gecelca y Celsia estaban en proceso de contratar comunicadores especializados en relaciones con la comunidad. El apoyo de los comunicadores a la responsabilidad social, como práctica corporativa, hacía parte, en estas dos organizaciones, del trabajo que realizaba el responsable del área de comunicaciones, quien se encargaba de la totalidad de la gestión comunicativa de la entidad. En la Empresa Urrá, la jefe de divulgación y prensa era la única persona que se ocupaba de las actividades especializadas en comunicación.

Al consultar por el papel que cumplen los profesionales de la comunicación en los programas de responsabilidad social, la totalidad de los entrevistados coincide en que la tarea es apoyar, porque, como se ha mencionado, esta es una labor a la que se articula el trabajo de distintas dependencias.

En lo que respecta al pensamiento alrededor del cual se gestiona la responsabilidad social, las empresas están convencidas de que esta no es un añadido a la operación, sino que hace parte de la concepción misma de cada entidad. Algunos la definen como la manera en la que se comporta el personal y la forma como hace el trabajo. Se habla de que incluye acciones voluntarias, pero también del cumplimiento de las obligaciones, porque, según dice uno de los entrevistados, en el contexto latinoamericano lo obligatorio todavía tiene muchos vacíos. En este sentido, una primera responsabilidad de la empresa es acatar la ley. Los requerimientos legales son importantes, porque en algunos casos la construcción de centrales hidroeléctricas ha supuesto la reubicación de comunidades indígenas y campesinas, lo que exige el cumplimiento de licencias ambientales y el pago de multas diferidas en el tiempo, a la par que la puesta en marcha de acciones de mitigación de los impactos en el medio ambiente y en los grupos sociales asentados en las zonas de influencia. 
Las concepciones están marcadas, también, por la noción del triple balance, razón por la que los entrevistados plantean que la responsabilidad social consiste en generar valor desde lo económico, lo social y lo medioambiental, en un marco de relación con todos los grupos de interés. Con un pensamiento similar, pero desde el concepto de sostenibilidad, EPSA concluye que tiene un compromiso simultáneo con el entorno y con los grupos de interés, a los que busca impactar desde cada una de sus líneas de negocio. Para esta organización, la empresa sostenible es aquella que se considera parte de la sociedad y que, en esa medida, es consciente de sus impactos sociales, económicos y ecológicos y se centra en agregar valor a dichas esferas. Desde sus formulaciones estratégicas, define la sostenibilidad como "una mesa de cuatro patas", donde cada una de ellas corresponde a un componente: económico, social, ambiental y de gestión humana.

Ya sea que se le denomine "responsabilidad social", "responsabilidad empresarial" o "sostenibilidad", la gestión de estas cuestiones incluye el gerenciamiento de los impactos positivos y negativos de las operaciones de la empresa; también es un gesto de solidaridad e implica la obligación de respetar los derechos de los ciudadanos.

\section{Relaciones públicas y gestión de la responsabilidad social}

Cuando se consulta si las organizaciones trabajan desde las relaciones públicas para gestionar la responsabilidad social, se encuentra: a) que tres comunicadores entrevistados juzgan las relaciones públicas a partir de prácticas y modelos anteriores al bidireccional simétrico; b) un profesional piensa que los profesionales del área deben prepararse para asumir el nuevo reto que representa la comunicación de la responsabilidad empresarial, sin agotarse en denominaciones profesionales; c) están, asimismo, las visiones de quienes consideran que las relaciones públicas se hacen presentes en todo el marco de interacciones con los grupos de interés, $\mathrm{y} d$ ) en general, se coincide en que el relacionamiento con los públicos, en la gestión de la responsabilidad social, no se orienta ni se realiza únicamente desde las áreas de comunicaciones. 
En cuanto a la primera consideración, está el punto de vista de quien afirma que en su empresa "las relaciones públicas no cumplen un papel de apoyo a la responsabilidad social para nada" (entrevista personal, mayo de 2012). Dice que la gestión de la sostenibilidad se hace, ante todo, de cara a las comunidades, a los organismos ambientales y a los gobiernos locales o regionales. "A ellos no les sirve que yo vaya con una estrategia de relaciones públicas. A ellos hay que llevarles una estrategia de inversión, de identificación de prioridades, de necesidades mutuas: por parte de la empresa, pero también por parte de ellos".

Se consulta, en este caso, si es posible concebir las relaciones públicas como la búsqueda de relaciones armónicas con todos los grupos de interés en un marco de satisfacción de intereses mutuos para ganar legitimidad como empresa y, en ese sentido, sostenibilidad en el largo plazo. La respuesta es que, vistas desde una búsqueda de beneficio mutuo, podrían aplicarse, pero que "si las relaciones públicas fueran un trabajo para entender el contexto y la cosmovisión, el concepto del mundo que tienen los públicos, en concreto las comunidades, sí se aplicarían”. A su juicio, las relaciones públicas no están concebidas de esa manera. Son una agenda que se establece a cierto nivel, con un alcance muy diferente y que solo se aplica en el relacionamiento con el gobierno, los clientes, las empresas del mismo sector y los medios de comunicación.

Está además la postura de dos entrevistados para quienes en la empresa las relaciones públicas no se abordan desde la perspectiva de la persuasión ni desde la creación de consenso. La razón que se argumenta es que en la empresa "la responsabilidad social no es accesoria ni cosmética. La organización comunica hechos y desempeño, y avanza hacia modelos conversacionales, no hacia modelos persuasivos" (entrevista personal, marzo de 2012). Uno de estos entrevistados hace saber que es el área de proyección corporativa la que maneja el tema de las relaciones públicas y no el comunicador que apoya la gestión de la responsabilidad social. Su labor, dice, no se enfoca desde "la lógica per se del lobby (... ) porque la empresa busca un equilibrio con sus grupos de interés desde el cumplimiento con sus compromisos, más que desde un enfoque del lobby para el relacionamiento, que es lo que conocemos de las relaciones públicas". 
Ambos profesionales coinciden en que las relaciones públicas están presentes en la gestión con la prensa, las campañas institucionales y los eventos. Atendiendo a los adjetivos que utilizan, su mirada tiende a ser negativa, por cuanto las asocian a la existencia y manejo de una agenda que la organización traza con ciertos públicos y la relacionan con nociones como "accesoria", "cosmética" o "lobby". Cabe anotar que este último se ha practicado en Colombia, muchas veces, entregando dinero a personas que ocupan niveles intermedios en cargos oficiales para favorecer decisiones que son del interés de la organización (Molleda \& Suárez, 2004). Tampoco las consideran un apoyo importante en la gestión de relaciones con las comunidades. Ahora bien, atendiendo a las afirmaciones de los mismos entrevistados acerca de que debe valorarse a cada grupo de interés por igual, no podría decirse que únicamente las comunidades merezcan ser tenidas en cuenta, según su visión del mundo o sus necesidades más específicas. Es decir, queda en cuestión por qué se manejan enfoques que parecen considerar al público en su esencia, en unos casos, pero en otros no.

Entre los entrevistados se ha presentado, de otra parte, una postura que puede considerarse más moderada; no obstante el profesional que la propone advierte sobre los peligros derivados de una mala concepción de las relaciones públicas cuando se trata de apoyar los temas de responsabilidad empresarial. Él enfatiza en las competencias del profesional, más que en la función o la disciplina de estudios donde se apoye para gestionar estas temáticas. Afirma, entonces, que las relaciones públicas son un instrumento para tender puentes. Considera que la gestión de la responsabilidad social es un tema muy nuevo en América Latina, donde apenas se está en proceso de entender lo que hay detrás de esa iniciativa, y que se debe evitar, mostrando que se hacen cosas, que se vuelva banal o se convierta en un asunto de marketing o de posicionamiento de la marca con grupos de interés. Propone que "la labor de relaciones públicas tendría que meterse de lleno a comprender la esencia de toda esta apuesta para poder trascender el asunto de la inmediatez, del esnobismo, de la moda, para no caer en esas trampas" (entrevista personal, marzo de 2012). Concluye que el aporte de la comunicación es supremamente valioso, pero a los profesionales les falta meterse, con mucho más compromiso, a comprenderlo y dominarlo. 
Figuran, por último, los puntos de vista de quienes tienen el mejor concepto sobre el papel que cumplen las relaciones públicas en los programas de responsabilidad social. Ellos aceptan que definitivamente existe un componente de esta disciplina en el trabajo de las comunicaciones para gestionar la responsabilidad social y la sostenibilidad, para buscar la armonía con el entorno y el relacionamiento a partir de intereses recíprocos, lo que hace ganar legitimidad. Entienden las relaciones públicas como una manera de establecer un contacto más directo con los grupos de interés y como herramientas necesarias en la construcción de sentido. Para una de las entrevistadas de este grupo, todo su trabajo consiste en las relaciones públicas, porque la organización necesita acercarse a numerosos grupos de interés. Las concibe como actividades que involucran a la alta dirección, pues, a su juicio, las áreas de comunicaciones practican las relaciones públicas para establecer vínculos a los que luego otros actores terminan de dar forma, como sucede al firmar un convenio o sellar una alianza.

\section{Conclusiones}

Los resultados de este estudio indican que, si bien en numerosos trabajos previos se aportan elementos sobre los beneficios que tendría la práctica de las relaciones públicas en el apoyo a los programas de responsabilidad social, ese sustento no siempre se hace efectivo en el sector analizado, en parte, porque algunos de los entrevistados tienden a equipararlas con la construcción de una agenda favorable a la organización.

Los resultados del presente estudio guardan relación con los realizados por Benn, Renier \& Pendleton (2010), según los cuales, a pesar de que la teoría de las relaciones públicas ha evolucionado, existen percepciones negativas de la responsabilidad social empresarial cuando se le vincula al ejercicio de aquellas. Como encontraron en sus investigaciones, el problema radica, muchas veces, en que numerosos líderes de las organizaciones ven a estos profesionales solo como una fuente de publicidad positiva. Ahora bien, un aspecto crítico en el caso que aquí se ha analizado es que son los propios profesionales de la comunicación los que tienen una idea negativa sobre las relaciones públicas. Como se ha encontrado, algunos de ellos las perciben como manejo de agenda, lobby con entes públicos y re- 
laciones con los medios. Esto lleva a inferir que su visión es negativa o que desconocen los modelos que amparan unas prácticas incluyentes y con una visión más ética.

Este resultado permite generar una hipótesis que deberá validarse en estudios posteriores. De este modo, si se tiene en cuenta el impacto que el entorno político, social y cultural tiene en la formación y en la práctica de las relaciones públicas, es preciso mirar de manera específica la profesión en Colombia, donde estas fueron implantadas por compañías multinacionales que, como parte de su gestión, integraban funciones y tareas comunicativas o contrataban los servicios de empresas especializadas (Molleda \& Suárez, 2004). A excepción de Brasil, donde las relaciones públicas están legitimadas en el mercado laboral y en el ámbito académico mediante la Ley 5377 de 1967, en los demás países de América Latina solo en los últimos años han empezado a existir ofertas de formación con énfasis en comunicación en las organizaciones (Ferrari \& Franca, 2011), sobre todo en la modalidad de posgrado.

Llama la atención que en algunas empresas consultadas se contrate a comunicadores para que se dediquen, de manera prioritaria, a las relaciones con la comunidad y que no se hable de que se requieran o existan profesionales expertos, que se ocupan de las relaciones con accionistas, inversores o proveedores, que serían otros grupos de interés relevantes en el sector. Como han advertido los entrevistados, la implantación de la responsabilidad social ha conducido a abandonar la idea de que solo se debe ser responsables con las comunidades, pero la disponibilidad de comunicadores enfocados de manera particular en estos colectivos, si bien no es negativa, sílleva a inferir que se les sigue concediendo un interés especial.

\section{Referencias}

Benn, S., Renier T. L. \& Pendleton, J. (2010). Public relations leadership in corporate social responsibility. Journal of Business Ethics, 96, 403-423. 
Bowen, S. A. (2008). A state of neglect: Public relations as 'corporate conscience' or ethics counsel. Journal of Public Relations Research, 20(3), 271-296.

Celsia (2012). Colinversiones. Informe anual 2011. Recuperado de http:// www.celsia.com/site/Portals/0/contenidos/documentos/inversionistas/informacion_financiera/informes_anuales/1-2011.pdf (Fecha de consulta: 27 de julio de 2013).

Cier (2011). Sostenibilidad y equidad: un mejor futuro para todos. Recuperado de http://hdr.undp.org/es/informes/mundial/idh2011/resumen/ (Fecha de consulta: 20 de julio de 2013).

Clark, C. E. (2000). Differences between public relations and corporate social responsibility: An analysis. Public Relations Review, 16(3), 363-380.

Contraloría General de la República de Colombia (2012a). Informe de Auditoría. Interconexión Eléctrica S.A.E.S.P. - Isa. Vigencia 2011. Recuperado de http://186.116.129.19/c/document_library/get_file?p_l_id $=28179552 \&$ folderId=75394654\&name=DLFE-48430.pdf $(\mathrm{Fe}-$ cha de consulta: 2 de agosto de 2013).

Contraloría General de la República de Colombia (2012b). Informe de Auditoría. Generadora y comercializadora de energía del Caribe S.A. E.S.P. - Gecelca S. A. E. S. P. Vigencia 2011. Recuperado de http://186.116.129.19/c/document_library/get_file?p_l_id= 74635732 \&groupId $=10136 \&$ folderId $=75394654 \&$ name $=D L$ FE-48428.pdf (Fecha de consulta: 27 de julio de 2013).

Cottle, S. (2003). News, public relations and power: Mapping the field. Londres: Sage.

Cutlip, S. \& Center, A. (1952). Effective public relations, pathways to public favor. Englewood Cliffs: Prentice Hall. 
Daugherty, E. L. (2001). Public relations and social responsibility. En Heat, R. L. \& Vásquez, G. (Eds.), Handbook of public relations (389-401). Los Ángeles: Sage.

Dawkins, J. (2004). Corporate responsibility. The communication challenge. Journal of Communication Management, 9(2), 108-119.

Dinero (2012, 4 de julio). Negocio millonario. Dinero. Recuperado de http://www.dinero.com/negocios/articulo/negocio-millonario/154535 (Fecha de consulta: 27 de marzo de 2013).

Empresa Urrá (2012). Balance General 2011. Recuperado de http:/ /www. urra.com.co/documentos/balGralURRA.pdf (Fecha de consulta: 28 de marzo de 2013).

EPSA (2012). Informe anual 2011. Recuperado de http://www.epsa.com. co/LinkClick.aspx?fileticket=tyI7xs41w84\%3d\&tabid=233\&m $\mathrm{id}=340$ (Fecha de consulta: 27 de julio de 2013).

Ferrari, M. A. \& Franca, F. (2011). Relaciones públicas. Naturaleza, función y gestión en las organizaciones contemporáneas. Buenos Aires: La Crujía Ediciones.

Freeman, R. (1984). Strategic management: A stakeholder approach. Boston: Pitman Publishing Inc.

Freeman, R. E. \& Phillips, R. A. (2002). Stakeholder theory: A libertarian defense. Business Ethics Quarterly, 12(3), 331-341.

Grunig, J. A. (Ed.) (1992). Excellence in public relations and communication management. Hillsdale, N. J.: Lawrence Erlbaum Associates.

Grunig, J. E. \& Hunt, T. (1984). Managing public relations. Forth Worth: Harcourt Brace. 
Grunig, J. E. \& Grunig, L. (1992). Models of public relations and communication. En Grunig, J. E. (Ed.), Excellence in public relations and communication management (285-325). Hillsdale, N. J: Lawrence Erlbaum Associates.

Grupo EPM (2012). Estados financieros consolidados a 31 de diciembre de 2011 y 2012. Cifras expresadas en millones de pesos colombianos. Recuperado de http://www.epm.com.co/site/portals/documentos/ inversionistas/2011/Nota_a_Estados_Financieros_Consolidados_2011.pdf (Fecha de consulta: 28 de marzo de 2013).

Isagen (2012). Informe financiero Isagen S.A.E.S.P. Recuperado de http://www. isagen.com.co/comunicados/INFORME_FINANCIERO_4T_11. pdf (Fecha de consulta: 28 de marzo de 2013).

Jelen, A. (2008). The nature of scholarly endeavors in public relations. En Van Ruler, B., Vercic, A. T. \& Vercic, D. (Eds.), Public relations metrics. Research and evaluation (36-59). Nueva York: Routledge.

Kim, S. \& Park, H. (2011). Corporate social responsibility as an organizational attractiveness for prospectives public relations practitioner. Journal of Business Ethics, 103, 639-653.

Molleda, J. C. \& Suárez, A. M. (2004). Challenges in Colombia for public relations professionals: A qualitative assessment of the economic and political environments. Public Relations Review, 31, 21-29.

Preciado, A. \& Guzmán, H. (2011). ¿Cómo utilizan la comunicación estratégica las organizaciones colombianas? Revista Dircom, 91, 31-35.

Sharpe, M. L. \& Pritchard, B. J. (2004). The historical empowerment of public opinion and its relationship to emergence of public relations as a profession. Tilson, D. J. \& Alozie, E. C. (Eds.), Toward the common good: Perspectives in international public relations (1436). Boston: Allyn and Bacon. 
Spangler, I. \& Donnalyn, P. (2011). Corporate social responsibility and the oil industry: Theory and perspective fuel longitudinal view. Public Relations Review, 37, 217-225.

UPME (2012). Informe mensual de evolución de variables de generación. Subdirección de Planeamiento Energético. Julio 2012. Recuperado de http://www.siel.gov.co/portals/0/Boletin\%20UPME\%20 Julio\%202012.pdf (Fecha de consulta: 27 de julio de 2013).

UPME-Ascodis (2011). Sector eléctrico nacional. Informe sectorial sobre la evolución de la distribución y comercialización de energía eléctrica en Colombia. Recuperado de http://www.siel.gov.co/LinkClick.asp $\mathrm{x}$ ?fileticket=L9AASwJjMz8\%3d\&tabid=58 (Fecha de consulta: 28 de marzo de 2013).

Van Ruler, B., Vercic, A. T. \& Vercic, D. (2008). Public relations metrics. Measurement and evaluation. An overview. En Van Ruler, B., Vercic, A. T. \& Vercic, D. (Eds.), Public relations metrics. Research and evaluation (1-18). Nueva York: Routledge.

White, C. \& Park, J. (2010). Public perceptions of public relations. Public Relations Review, 36, 319-234. 